\title{
Accesibilidad e inclusión en espacios de arte: ¿cómo materializar la utopía?
}

\section{Accessibility and Inclusion in Art Spaces: How to realize the utopia?}

\author{
Luisa María Gómez del Águila \\ Universidad de Málaga. Departamento de Didáctica de la Expresión Musical, Plástica y Corporal \\ lmdelaguila@uma.es
}

Recibido: 28 de enero de 2011

Aprobado: 10 de mayo de 2011

\section{Resumen}

El derecho de acceso al medio, amparado por leyes nacionales e internacionales de todo rango, comienza a reconocerse a partir de las dos guerras mundiales y de la proclamación, en los años sesenta, del Principio de Normalización. A pesar de las décadas transcurridas, el imaginario colectivo continúa reduciéndolo a la accesibilidad física y sensorial, y obvia otros aspectos esenciales, que solo reciben cierta consideración entre especialistas.

A la par, las intervenciones institucionales en este sentido impulsan cambios en el medio físico o en los canales de comunicación, pero ignoran cuestiones fundamentales -como la accesibilidad conceptual-, especialmente significativas en contextos culturales. La situación se agrava en los espacios dedicados al arte, donde el mundo de los significados, que nos constituye como seres humanos, cobra especial relevancia. La falta de un corpus de conocimiento sobre la cuestión, la dispersión bibliográfica y la exigua atención que la literatura especializada presta a las barreras cognitivas, cierran el círculo.

Este artículo plantea una concepción más amplia de las barreras que intervienen en la exclusión, aborda la accesibilidad conceptual, y propone un modelo básico para futuras intervenciones, que recoge de forma coherente los diferentes aspectos que afectan a la inclusión en espacios de arte.

Palabras Clave: Arte e inclusión, proyectos de inclusión, accesibilidad, barreras físicas, sensoriales, cognitivas y sociales.

Gómez del Águila, L. 2011: Accesibilidad e inclusión en espacios de arte: ¿cómo materializar la utopía? Arte, Individuo y Sociedad, 24(1), 77-90

\begin{abstract}
Under a range of national and international laws, the right to access the medium is starting to be recognized since both World Wars and the proclamation of the Normalization Principle in the sixties. Despite the decades elapsed, the collective imagination continues reducing it to physical and sensory accessibility and ignores other essential aspects, only considered among specialist.

At the same time, institutional interventions promote changes in the physical medium or in the channels of communication, but ignore fundamental questions -like conceptual accessibility- particularly significant in cultural contexts. The situation is exacerbated in spaces dedicated to art, where the world of meanings (that make us humans) takes special relevance. The circle is closed by a lack of a body of knowledge about the question, the bibliographic dispersion and the meagre attention that specialist literature gives to cognitive barriers.
\end{abstract}


This article presents a wider conception of barriers that take part in exclusion. It addresses conceptual accessibility and proposes a basic model for future interventions that can capture coherently the different aspects that affect inclusion in art spaces.

Key Words: Arts and inclusion, inclusion projects, accessibility; barriers physical, sensory, cognitive and social.

Gómez del Águila, L. 2011: Accessibility and Inclusion in Art Spaces: How to realize the utopia? Arte, Individuo y Sociedad, 24(1), 77-90

Sumario: 1. De la segregación establecida a la normalización teórica, 2. ¿Espacios de arte ...inclusivos?, 2.1 Una oferta segregada..., 2.2 ...y centrada en las barreras más evidentes, 3. ¿Qué, cómo, cuándo?,

3.1 Barreras, 3.2 Proyectos de inclusión en espacios dedicados al arte, 4. Inclusión, ¿para quién?. Referencias

El acceso al medio es un derecho básico de cualquier ser humano, reconocido por leyes nacionales e internacionales que, hasta el momento, ni siquiera los países que se consideran más avanzados han logrado garantizar. Por el contrario, los índices objetivos de discriminación que en este sentido soportan ciertos colectivos son muy elevados en comparación con los que soporta la sociedad en su conjunto (Congreso Europeo, 2002). En consecuencia, la aproximación de muchas personas al mundo físico y cultural del que forman parte sigue dependiendo, en gran medida, de variables sobre las que individualmente solemos tener poco o nulo control: procedencia sociocultural, nivel económico, contexto en el que nos desarrollamos, aprendizajes previos... y, también, esas peculiaridades que hemos dado en llamar "discapacidades" (Gómez del Águila, 2003).

Esta situación, que conlleva graves consecuencias para diferentes sectores de la población, supone un déficit social difícilmente justificable, al afectar tanto al desarrollo personal de muchos seres humanos -que ven limitadas sus posibilidades de acceso al conocimiento-, como a su participación -al dificultar el acceso a situaciones habituales en la sociedad a la que pertenecen-. Numerosas barreras físicas, sociales y actitudinales impiden a muchas personas participar en igualdad. Derribarlas y ofrecer contextos ricos en estímulos y oportunidades diversas de establecer contacto con la sociedad (Cuadrado, 1996: 185), requiere un cambio de mentalidad que desplace el énfasis de las carencias y dificultades a las potencialidades presentes en cualquier ser humano (Kaushik, 2000; Gómez del Águila, 2003). Es necesario trasladar el foco de lo individual a lo colectivo.

Este cambio, urgente en lugares donde predomina su uso práctico -calles, transporte público, comercios, hospitales...-, aún lo es más en aquellos donde el mundo de los significados cobra especial relevancia; en espacios que, más allá de la realidad objetivable, nos permiten conformarnos como verdaderos seres humanos (Vigotsky, 1987). Así, es fundamental que los espacios culturales -con su máxima expresión en los centros de arte, que propician tanto la apreciación como la producción de obra-, inicien con urgencia el camino hacia la inclusión. 


\section{De la segregación establecida a la normalización teórica}

La conciencia de dicha necesidad hunde sus raíces en los años posteriores a las dos guerras mundiales, cuando, debido a la unión de los avances armamentísticos y de la medicina, sobreviven muchos combatientes que presentan importantes secuelas. Este aumento de hombres jóvenes con dificultades para interactuar en diferentes ámbitos, traslada a la esfera pública una situación hasta entonces considerada privada: hasta el momento, las personas con algún tipo de discapacidad hacía una vida sumamente reservada, solo en contacto con sus familiares más directos.

Es decir, en este momento, el núcleo del problema se desplaza del individuo al entorno (Gorbeña, González y Lázaro, 1997) y las sociedades occidentales comienzan a plantearse la participación sociocultural de quienes, en algunos aspectos, se desenvuelven de manera diferente a la mayoría. Las primeras actuaciones son de carácter ocupacional y rehabilitador (DeJong 1979, 1981), y subrayan la diferencia, generando propuestas específicas y bien diferenciadas de las del resto del público.

Sin embargo, en el plano teórico esta lógica va perdiendo terreno poco a poco a favor del Principio de Normalización (Nirje, 1969; Bank-Mikkelsen, 1975; Wolfensberger, 1972). Aunque proclamado en el contexto de las personas consideradas con déficit intelectual, en la actualidad se extiende a toda la población: no hay personas diferentes, todas y todos lo somos. Desde este punto de vista, la sociedad es entendida como una realidad múltiple y diversa, formada por infinitas variantes personales que, al interactuar, se enriquecen entre sí. En otras palabras: sobre el papel las distintas realidades individuales, asumidas como diferentes posibilidades de realización personal, se sitúan en idéntico plano de importancia. En este marco, las personas con hándicap no son sino personas normales, que quieren hacer cosas normales, aunque el orden social se lo dificulta (King, 1996).

De acuerdo con ello, los programas de los espacios culturales deberían ser accesibles para un amplísimo abanico de visitantes, dando respuesta a muchas peculiaridades en la actualidad ignoradas o relegadas. En el marco del Principio de Normalización, la tradicional separación "público en general-personas con necesidades especiales", no tiene cabida. Por tanto, la dicotomía entre una oferta general para visitantes-tipo, y otra alternativa para quienes no encajen en dicho patrón, carece de sentido.

\section{2. ¿Espacios de arte ...inclusivos?}

En los últimos años, los museos y centros de arte, inicialmente diseñados para un perfil muy concreto de visitante, empiezan a tener en consideración cuestiones de accesibilidad. Sin embargo, un análisis detenido de su oferta, evidencia la hegemonía del modelo tradicional segregado o, en el mejor de los casos, integrador, aún muy alejado de un modelo inclusivo. De este modo, y bajo una apariencia de oferta abierta, se siguen soslayando las condiciones que favorecen una experiencia eficaz, confortable y autónoma para cualquier persona, con independencia de sus peculiaridades. En esta situación, ni siquiera una gran motivación -a menudo minada por múltiples experiencias frustrantes (Schouten, 1994)-, es suficiente para salvar los obstáculos que dificultan, a una parte importante del público -no solo a personas con discapacidad-, disfrutar de su oferta. 
La situación se agrava cuando dichos espacios se promocionan como accesibles, con la consiguiente frustración de quienes acuden a ellos -a veces, realizando un gran esfuerzo personal- con la esperanza de que lo sean.

Dichas trabas se apoyan en dos prácticas todavía habituales: por un lado, una oferta segregada, que relega a un segundo plano las necesidades de quienes no encajan en el patrón ideal de visitante. Por otro, propuestas que abordan la accesibilidad física $\mathrm{y}$, en algunos casos, sensorial, pero ignoran tanto las barreras cognitivas -con un alto grado de incidencia en la accesibilidad del público en general-, como las sociales, en la base de todas las anteriores.

\subsection{Una oferta segregada...}

Basta repasar la información que ofrecen al público -in situ o a través de sus páginas web- los museos y centros de arte más emblemáticos de nuestro país, para comprobar lo habitual de esta práctica: la oferta se segrega en función de las características del público que tiene en consideración. Así, las visitas generales se separan de las de visitantes con ciertos tipos de hándicap -generalmente, dificultades motoras y sensoriales y, en los últimos años, con enfermedades mentales-, y queda fuera un tercer grupo, cuyas peculiaridades no encajan en los patrones más conocidos (por ejemplo, personas con sordoceguera, dificultad para acceder a contenidos abstractos o un nivel sociocultural considerado por debajo de la media).

Esta situación se complica porque, mientras la oferta general es prácticamente diaria (museos y otros espacios de arte suelen estar abiertos seis días semanales) y de toda la obra expuesta, la oferta para personas con hándicap es mucho más reducida, tanto en el número de piezas o exposiciones accesibles como en las fechas en que es posible la visita, limitándose en ocasiones a un único día semanal, quincenal y, en algunos casos, mensual. A esto hay que sumar que los requisitos en caso de estos recorridos son, a menudo, más estrictos que los que implica la visita programada con carácter general: número mínimo de participantes, reservas con mayor antelación, etc., que suponen una traba más o menos importante en función de diferentes cuestiones, como el grado de dependencia de cada persona o el peso de anteriores experiencias en espacios de este tipo.

\section{2 ...y centrada en las barreras más evidentes}

La cuestión todavía se complica porque es habitual que las propuestas denominadas "accesibles" aborden la accesibilidad física y, en el mejor de los casos, sensorial, pero ignoren tanto las barreras cognitivas -con un alto grado de incidencia en la accesibilidad del público en general y de los colectivos que habitualmente quedan fuera de dicha consideración-, como las sociales, a pesar de que están en la base de todas las demás. Incluso jornadas especializadas en arte, accesibilidad y diseño universal, organizadas en el marco de instituciones de primer orden, evidencian dicha tendencia: "El cuerpo de la letra, la tipografía, las cartelas, la altura de las vitrinas o el contraste de colores son algunas de las cosas que tenemos que tener presentes al diseñar y encargar una exposición" (Disseny Hub Barcelona, 2010) 
En estas condiciones, cualquier diferencia personal que exceda límites muy precisos, se presenta cargada de connotaciones negativas -"no soy capaz de..., yo no valgo para esto..., esta gente no puede..."- y no es posible hablar de inclusión. Quienes gestionan el patrimonio, eluden así uno de los compromisos éticos esenciales que implica dicha responsabilidad: ponerlo al alcance de toda la sociedad.

\section{3. ¿Qué, cómo, cuándo?}

La necesidad de atender cuanto antes dicho compromiso es evidente. Pero también lo es la dificultad que las instituciones están teniendo para hacerlo. En última instancia se alegan desde cuestiones referentes a la conservación, hasta limitaciones presupuestarias. Sin embargo, antes de enfrentarnos a la constatación de dichos problemas -o la búsqueda de soluciones, cuando exista un choque de necesidades real-, es preciso revisar la concepción de las barreras que limitan la oferta de los espacios de arte, y asumir que para lograr la inclusión, no es suficiente con eliminar de forma parcial las más conocidas. Cuanto más tiempo dure la transición, más personas verán limitados sus derechos.

\subsection{Barreras}

A lo largo de las últimas décadas se han identificado barreras de diferentes tipos -físicas, urbanísticas, arquitectónicas, del transporte, sensoriales, de comunicación e información, culturales, psicológicas, emocionales...- que dificultan la aproximación a espacios, piezas o documentos, la comprensión de su sentido o ambos aspectos, por lo que entorpecen la participación y el acceso al conocimiento de muchas personas.

Un obstáculo básico para la erradicación de dichas barreras es la ausencia de una clasificación generalmente aceptada, que pueda servir de base a una concepción de las mismas más amplia y coherente con la realidad. Esta situación, que se enmarca en la ausencia de un corpus teórico sobre la cuestión, impide que las intervenciones en espacios de arte tengan carácter inclusivo, ya que dejan fuera de la actuación las barreras menos evidentes, especialmente significativas en estos lugares.

De hecho, las barreras cognitivas son habitualmente olvidadas en la literatura especializada y, en consecuencia, en los espacios patrimoniales. Es especialmente complejo el caso de los espacios de arte, lenguaje a menudo considerado universal e imprescindible para el desarrollo humano que, sin embargo, sigue estableciendo entre obra y público con discapacidad intrincadas barreras excluyentes (Díez, 1996). De acuerdo con ello, deben ser consideradas las siguientes barreras:

- Barreras sociales.

- Barreras para la accesibilidad física.

- Barreras sensoriales.

- Barreras cognitivas. 


\subsubsection{Barreras sociales}

En la base de todas las demás, retroalimentan la vigencia de paradigmas teóricamente superados. Se basan en la comparación de cada persona con un modelo de ser humano ideal, e inexistente. Cuanto más se aleje alguien de dicho estándar, más negativa será la consideración que la sociedad le otorgue y, en consecuencia, más trabas tendrá que superar para participar. Dicha desconsideración es a veces muy sutil $\mathrm{y}$, en ocasiones, bienintencionada, lo que complica aún más la cuestión (Congreso Europeo, 2002). Pero también puede ser muy burda, como cuando ante la demanda de una usuaria, el guarda situado en una puerta grita a su compañero, al otro lado de un patio: “¡Pepeee, abre el portón, que va una silla!”.

Las barreras sociales implican la exclusión porque, de modo más o menos encubierto y siendo o no conscientes de ello -tanto la institución como quienes la visitan-, las personas que no encajan en lo que entendemos por "normal" quedan fuera del público objetivo para el que se diseña un espacio o actividad. Es decir, mientras en teoría se potencia y valora la participación en actos sociales y culturales, en la práctica asociaciones e instituciones ponen trabas al acceso a sus actividades de personas con capacidades diferentes (Alberich, Aldaz y Fernández, 1996).

Esto ocurre cuando un museo evidencia que realiza su oferta para un "visitantetipo" y, en el mejor de los casos, adopta medidas de carácter "paliativas", para contrarrestar esta posición de partida: entradas accesibles que constituyen verdaderas puertas secundarias; aseos para hombres, mujeres y personas con movilidad reducida; aseos para personas con movilidad reducida cerrados con llave; insistencia del personal en ofrecer ayudas que el usuario o usuaria no necesita; uso de un lenguaje discriminatorio -disminuidos, minusválidos, deficientes- o infantilizado; folletos con fotos de visitantes sólo sin discapacidad; oferta de actividades accesibles, pero diferenciadas de las del público en general -que, además, son una disyuntiva para sus acompañantes- o limitada a un número inferior de sesiones o a una pequeña parte de la muestra; etc.

\subsubsection{Barreras para la accesibilidad física}

Aunque en el imaginario colectivo suelen aparecer como elementos que impiden el paso o el acercamiento a la obra a quienes tienen dificultades de movilidad -es decir, como barreras físicas-, su definición es mucho más amplia: una barrera para la accesibilidad física es cualquier elemento espacial, localizado en cualquier punto del recorrido necesario para disfrutar del espacio -por ejemplo, la cafetería, la tienda o los aseos de un museo- que, por deficiencias formales, de ubicación o de material, dificulta el uso seguro, confortable, eficaz y normalizado de sitios y objetos.

Son barreras para la accesibilidad física escaleras y rampas con excesiva pendiente; mostradores, perchas, teléfonos... situados a mucha altura; elementos colgantes por debajo de 2,20 m; puertas estrechas; etc. Pero también, pavimentos deslizantes o en mal estado; vidrios no señalizados o mal señalizados, pomos de difícil agarre o manipulación; vitrinas u otro mobiliario con aristas y cantos no redondeados; ilumi- 
nación escasa o deslumbrante; falta de asientos para el descanso durante el recorrido; etc. (Grosbois, 1994)

\subsubsection{Barreras sensoriales}

Son características del medio que dificultan la percepción. Aparecen:

- Cuando no se ha previsto alguno de los tres canales perceptivos -vista, tacto, oído- que nos permiten comunicarnos. Por ejemplo, cuando las cartelas no aparecen en braille, no existen réplicas táctiles de las obras, los avisos sonoros no pueden verse también en paneles visuales, etc.

- Si los mensajes presentan deficiencias formales, de contraste, o de tamaño o intensidad que impiden o limitan su percepción: imágenes y textos diminutos, escritos con tipos poco legibles o que apenas resaltan sobre el fondo; comunicaciones que no se entienden por el ruido ambiente o la reverberación de la sala, etc.

\subsubsection{Barreras cognitivas}

O deficiencias en la construcción del mensaje que entorpecen su comprensión. En este caso, el código se percibe, pero el mensaje no se entiende o exige gran esfuerzo cognitivo. Aparecen cuando el diseño de la información no respeta las condiciones de recogida y asimilación de datos del ser humano, y se deben tanto a fallos en la organización del espacio y su contenido, como en el diseño de los mensajes verbales o icónicos. Afectan a:

- Información instrumental, es decir, los datos que hay que conocer sobre la mecánica de la actividad para desenvolverse eficazmente en ella.

- Información espacial: cómo es éste sitio y cómo se llega -¡e identifica!- cada una de sus partes.

- Datos conceptuales: qué hay aquí, cual es su valor, porqué esto es importante, porqué se considera bello y, sobre todo, qué tiene que ver conmigo.

Existen barreras cognitivas en espacios de arte, cuando:

- Falta información sobre horarios, normas, etc.

- La organización de espacios, piezas o datos presenta incoherencias.

- No hay planos o señalización disponibles o, los que hay, presentan deficiencias de diseño que afectan a su comprensión.

- Hay enunciados -verbales o icónicos- que ignoran las particularidades del aprendizaje en función de las diferentes peculiaridades.

- Los nuevos conocimientos no se apoyan en el conocimiento del público o no se dan las condiciones que permitan relacionarlos con él.

- Hay contradicciones entre diferentes mediadores. 
- La información es pobre, excesiva o no diferencia niveles. Los mensajes son poco precisos.

- Se utilizan frases largas o vocablos poco familiares en textos que deben ser facilitadores (paneles, folletos, etc.). Las palabras clave se sitúan al final de la frase, dificultando su recuerdo.

- Se emplean términos técnicos sin ofrecer su explicación (Richadeau, 1976; López, 1982; Comes, 1992)

A pesar de su trascendencia, estas barreras apenas se tienen en cuenta y suelen enmascararse con explicaciones poco realistas, que trasladan la responsabilidad de la comunicación al público y tienen un efecto muy desmotivador. A veces, cuando visitamos un espacio patrimonial no comprendemos que no se ha producido el acercamiento personal necesario, o no hemos captado su mensaje -como al valorar una pintura en función de su parecido con la realidad-; otras, somos conscientes pero, en vez de buscar explicación en un hecho externo -mal diseño del mensaje o del mediador que nos debería facilitar el acercamiento-, creemos que nuestras capacidades personales no bastan para entenderlo. Quizá una de las evidencias más claras sea la dificultad del público actual para vivenciar gran parte de la obra creada a partir de las vanguardias del siglo XX.

Tanto las barreras sensoriales como las cognitivas interfieren en la comunicación, y su presencia impide la experiencia del arte.

\subsection{Proyectos de inclusión en espacios dedicados al arte}

Que niñas, niños y personas mayores, quienes no hablen el idioma mayoritario, procedan de culturas diferentes a la hegemónica, hayan desarrollado diferentes capacidades... gocen de las mismas oportunidades de conocimiento y disfrute en los espacios culturales que el resto de visitantes, depende del grado de inclusión existente en ellos. Los proyectos de inclusión, eliminando impedimentos y potenciando posibilidades, optimizan el acceso de todas y todos a los elementos del espacio físico, contenidos y experiencias posibles en cada lugar.

Dichos proyectos son especialmente relevantes en los espacios artísticos, que nos permiten el contacto más sublime con el mundo de sentidos y significados sin el cual, el ser humano, no puede considerarse como tal (Vigotsky, 1987). Pero mientras en ámbitos que podríamos llamar funcionales -calles, tiendas, hospitales-, lo esencial es resolver la comprensión y uso eficaz del espacio y sus elementos -o, dicho de otro modo, de la realidad evidente, la que percibimos con nuestros sentidos- en los espacios patrimoniales, además, hay que posibilitar el acceso a experiencias y conceptos de diferente índole, interrelacionados y situados a varios niveles de abstracción -a veces, muy elevados-, de modo que puedan experimentarlas y entenderlos, con el menor esfuerzo posible, personas con dificultades en diferentes áreas y en diferentes momentos de su desarrollo.

Esto implica resolver de forma adecuada la accesibilidad física, sensorial y cognitiva, y ello pasa por cuestionar profundamente los planteamientos sociales que están 
en su base. A partir de ahí, comienza un diseño de esquemas de circulación y zonificación coherentes, que faciliten:

- La movilidad independiente.

- La orientación espacial.

- El acceso a cada elemento.

- La comprensión del mensaje expositivo.

- Las vivencias personales en torno a la obra.

Cuando, como pasa con frecuencia en espacios históricos y naturales, no sea posible ni eliminar ni salvar con ayudas técnicas las barreras que limitan el acceso físico, debemos compensarlas con materiales que permitan entender $-y$, en la medida de lo posible, experimentar- el elemento al que no se puede llegar: réplicas, maquetas, ilustraciones, textos, grabaciones...

\subsubsection{Accesibilidad social}

Las intervenciones de todo el personal de la institución deben ajustarse a una idea del ser humano coherente con un planteamiento de carácter inclusivo, que entienda la necesidad de normalizar la presencia de personas diferentes en cualquier tipo de espacio. Para ello, habitualmente será necesaria una formación específica y continuada que ayude arroje luz y permita cuestionar ideas previas teóricamente superadas, pero aún en la base de la mayoría de las prácticas institucionales (Aguado, Flórez y Alcedo, 2001; Arte, 2001)

\subsubsection{Accesibilidad física y sensorial}

Asegurar tanto la accesibilidad física como la accesibilidad sensorial, exige un diseño espacial y de elementos que respete todos y cada uno de los principios del Diseño Universal:

1. Uso equiparable. Deben proporcionarse formas de utilización idénticas o equivalentes a cualquier visitante, evitando segregar a parte del público. La concreción de los elementos debe resultar atractiva para personas con diferentes peculiaridades.

2. Uso flexible, acomodable a un vasto abanico de preferencias y habilidades individuales. Debe facilitarse la exactitud y la precisión, y adaptarse al paso o ritmo de quien lo utiliza.

3. Uso simple e intuitivo, sin complejidad innecesaria. Consistente con las expectativas e intuición de quien lo use, y acomodable a un amplio rango de alfabetización y habilidades lingüísticas. La información debe dispensarse de modo coherente con su importancia, mediante sistemas de comunicación eficientes.

4. Información perceptible. El diseño de cualquier elemento debe comunicar con eficacia los datos necesarios para su manejo, atendiendo a las condiciones am- 
bientales y presentando la información esencial de modo gráfico, sonoro y táctil. Tiene que ser compatible con los dispositivos que utilizan las personas con discapacidad sensorial.

5. Tolerancia al error. Los riesgos y consecuencias negativas de errores o accidentes deben ser mínimos, y advertirse con eficacia.

6. Poco esfuerzo físico. El uso de los elementos debe ser eficaz y confortable, rentabilizando las fuerzas necesarias. Deben permitir una posición corporal neutra, y minimizar las acciones repetitivas y el esfuerzo físico continuado.

7. Tamaño y espacio necesarios. Su empleo tiene que ser posible y confortable para diferentes tamaños corporales, posturas, niveles de movilidad y usos de ayudas técnicas o de asistencia personal (Connell et. al., 1997).

Todo ello respetando la necesidad, fundamental en muchos espacios patrimoniales, de preservar sin modificaciones el medio y, por supuesto, la colección.

\subsubsection{Accesibilidad cognitiva}

Implica la ausencia de barreras para la comunicación. Pasa por un tratamiento global de la información que atienda tanto a la selección y ubicación de elementos expuestos, como a los mediadores que ayudarán a entender su sentido. Para ello, es necesario:

- Seleccionar los datos imprescindibles, tanto de carácter instrumental, como espacial y conceptual. Una selección ajustada facilita la visita de todo el público y minimiza la sobrecarga de la memoria a corto plazo que presentan personas con ciertas peculiaridades, como la ceguera, que obliga a recordar los muchos datos siempre disponibles para quienes ven.

- Estructurar claramente las ideas. Hay que decidir cómo separarlas, en qué orden ofrecerlas y cómo establecer -de modo explícito o no- las interrelaciones que deben darles coherencia. Este punto, junto con el anterior, es elemental para mejorar la visita de personas con escasa capacidad de concentración, coordinación y memorización, y dificultades de ubicación espacio-temporal y comunicación. Sin embargo, a menudo visitantes-tipo tienen dificultades debido a una selección de datos excesiva o una estructuración poco clara de los mismos.

- Determinar el código más adecuado para transmitir cada tipo de datos: texto, imagen plana, maqueta, réplica, audiovisual...

- Precisar los canales perceptivos que se utilizarán en cada caso, sabiendo que toda la información debe estar disponible para más de uno y, los datos fundamentales, para los tres sentidos básicos para la comunicación (Dischinger, 2000).

- Considerar las especificidades de comprensión de ciertos conceptos que habitualmente presentan quienes tienen ceguera o sordera, bien por tratarse de conceptos de carácter sensorial -como muchos de los relacionados con el arte-, bien por una falta de aprendizaje con referentes reales que dificulta el establecimiento de relaciones código-referente (Consuegra, 2000, 2001). 
- Por último, dar forma -redactar textos, crear imágenes, diseñar paneles...-, teniendo en cuenta las pautas que fundamentan tanto la legibilidad -o facilidad con la que puede percibirse el mensaje- como la lecturabilidad $-o$ facilidad con la que puede entenderse su sentido- (Barker y Fraser, 2000; Bellini, 2000). Es importante tener presente que la mera presentación de las cosas (...) mediante fotografías, dibujos, modelos o la exhibición en vídeo, no es garantía de la captación comprensiva del tema (Arnheim, 1986: 320) y que, en consecuencia, las imágenes, para ser accesibles, también deben respetar pautas de accesibilidad (Gerson y Hooper, 2000)

Todo ello, estableciendo relaciones coherentes y explícitas entre espacios, mediadores y contenidos. Se trata, en resumen, de realizar un esfuerzo de comunicación global, no excluyente, capaz de resolver los desajustes que pudieran producirse y que generalice una experiencia autónoma, activa y centrada en la percepción individual. $\mathrm{O}$, dicho de otro modo, se trata de adecuar la oferta a la persona y no la persona a la oferta.

Ahora bien, si resolvemos de forma óptima cada uno de los aspectos anteriores, ¿todo será accesible para todas las personas? La pregunta tiene una doble vertiente. Si hablamos de acceso físico la respuesta es negativa: en espacios patrimoniales generalmente prevalecen criterios de conservación y, en muchos sitios, habrá limitaciones objetivas que impidan, por ejemplo, que quienes tienen movilidad reducida entren en una alcazaba, que pueda tocarse una estatuilla original o que una persona con ceguera acceda a la experiencia pictórica que sólo puede generarse en contacto con la obra original. Ahora bien, sí será posible garantizar el acceso conceptual, de manera que cualquier persona, lo más cerca posible de los originales, pueda entender su valor y vivir una experiencia lo más cercana posible a la que estos pueden proporcionar.

\section{Inclusión, ¿para quién?}

La idea de inclusión supera a la de accesibilidad entendida como solidaridad con determinados colectivos. De hecho, cuando se actúa de forma aislada sobre necesidades específicas suelen crearse nuevas barreras que afectan no sólo a grupos con otras dificultades sino, también, al grueso de la población (Gómez del Águila, 2003; Rodríguez de Luengo, 1999).

Por eso, aunque conceptos como accesibilidad e inclusión nacieron para responder a las necesidades de las personas que, por diferentes causas, no podían acceder al disfrute de algunos derechos generalizados para el resto, en la actualidad los proyectos de inclusión en espacios dedicados al arte, además de crear puntos de encuentro en igualdad y normalizar la coexistencia de públicos diversos, tienen el objetivo de mejorar la visita de todas las personas, porque:

- Disminuyen los accidentes y el cansancio.

- Rentabilizan el esfuerzo cognitivo: se aprenden más cosas. La exposición se entiende y recuerda mejor. 
- Permiten entrar en contacto con lenguajes diferentes y disfrutar de sentidos casi olvidados en nuestra cultura, fuente de aprendizaje, experiencias poco habituales y, generalmente, placer.

- El público se siente competente y protagonista de la actividad, y tiende a establecer lazos afectivos con ella.

- Su carácter lúdico y distendido favorece nuevas visitas, al mismo o a otros espacios patrimoniales en general, y artísticos, en particular.

- Ofrecen la oportunidad de compartir tiempos y espacios con personas diferentes. Y esto ayuda a adquirir una visión de la sociedad más real, alejada de estereotipos que nos adjudican roles en función de criterios preestablecidos.

Por eso, al hablar de proyectos de inclusión en espacios de arte, no hablamos de proyectos para determinadas personas; hablamos de una oferta que permita, a cualquiera que lo desee, acceder a la propuesta en condiciones óptimas de seguridad, confort y eficacia. Es decir, hablamos de poner el arte, por fin, al alcance de cualquier visitante.

\section{Referencias}

Aguado, A.L., Florez, M.A. y Alcedo, M.A. (2001). Una estrategia de intervención y unos programas de cambio de actitudes ante la discapacidad. En: Verdugo

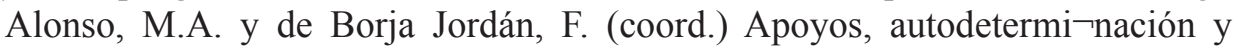
calidad de vida. Salamanca: Amarú.

Alberich, T., Aldaz, J. Y Fernández, Y. (1996). Orientación y movilidad con deficientes mentales. En: Actas del Congreso Estatal sobre Prestación de Servicios para Personas Ciegas y Deficientes Visuales: Madrid, septiembre de 1994, V, 116120. Madrid: ONCE.

Gerson, P. Y Hooper, V. (2000) Art History Through Touch and Sound. A Multisensory Guide for the Blind and Visually Impaired. Nueva York: An Art Education for the Blind, Optical Touch Systems y American Prin $\neg$ ting House fot the Blind.

Arte. Arte a portata di mano. Corso di formazione e aggiornamento per volontari che operano nel settore delle visite guidate tattili per non vedenti. (2001) Firenze: Volontari Associati per i Musei Italiani.

Barker, P. Y Fraser, J. (2000) Sign Design Guide: A guide to Inclusive Signage. London: Joint Mobility Unit Access Partnership.

Bellini, E. (2000) Toccare l'arte: L'Educazione estetica di ipovedente e non vedenti (comp.). Roma: Armando Armando.

Comes Nolla, G. (1992). Lectura y libros para niños especiales. Barcelona: Ceac.

Congreso Europeo sobre las Personas con Discapacidad (2002). Declaración de Madrid: No discriminación más acción positiva es igual a inclusión. http://www. eurociu.org/documentos/declamadrid_es.pdf (Fecha consulta: 18-11-2010). 
Connell, B.R., Jones, M., Mace, R., Mueller, J., Mullick, A., Ostroff, E. Y Sanford, J. (Comp.) (1997). Los principios del Diseño Universal. Steinfeld, Molly Story \& Gregg Vanderheiden.

Consuegra Cano, B. (2000). Comunicación y visitantes con discapacidad visual en espacios expositivos. Boletín ANABAD, XLX, 1, 143-157.

Consuegra Cano, B. (2001). El acceso al patrimonio histórico de las personas ciegas $y$ deficientes visuales. Madrid: ONCE.

Cuadrado Gomis, J. (1996). El ámbito residencial de la educación residencial. En: Actas del Congreso Estatal sobre Prestación de Servicios para Personas Ciegas y Deficientes Visuales: Madrid, septiembre de 1994, III, 181-190. Madrid: ONCE. Dischinger, M. (2000) Designing for all Senses: Accessible Spaces for Visually Impaired Citizens. Göteborg: Chalmers University of Technology.

Federación Española de Municipios y Provincias (1991). La Administración Local en la supresión de barreras arquitectónicas. Madrid: FEMP.

Fernández García, T., González Hernández, E., Bravo Sánchez, M.A. Y Galván Domínguez, F. (1996). Adecuación de ambientes fisicos. Experiencias de colaboración de la Unidad con entidades ajenas a la ONCE. En: Actas del Congreso Estatal sobre Prestación de Servicios para Personas Ciegas y Deficientes Visuales: Madrid, septiembre de 1994, V, 198-201. Madrid: ONCE.

Gómez del Águila, L.M. (2003). La Alhambra a ciegas: propuesta de un método de comunicación del entorno abierto a personas con ceguera o deficiencia visual. Tesis doctoral: Granada.

Gorbeña Etxebarría, S., González Prieto, V.J. Y Lázaro Fernández, Y. (1997). El Derecho al Ocio de las personas con discapacidad. Bilbao: Universidad de Deusto.

Grosbois, L.P. (1994) Ergonomía y museología. En: Foundation de France-ICOM. Museos abiertos a todos los sentidos. Acoger mejor a las personas minusválidas, 81-86. Madrid: Ministerio de Cultura y ONCE.

Kaushik, R. (2000) Acceso denegado: ¿podemos superar nuestra actitud ante los impedidos? Museum Internacional, 203 (51-3), 48-52.

King, S.P. (1996) El impacto de la tecnología en el acceso a la información y la cultura: la perspectiva del RNIB. En: Actas del Congreso Estatal sobre Prestación de Servicios para Personas Ciegas y Deficientes Visuales: Madrid, septiembre de 1994, I, 137-144. Madrid: ONCE.

Lavado Paradinas, P.J. (1991) El museo (im)posible: un museo funcional para visitantes especiales. En: VIII Jornadas Estatales DEAC Museos, 35-40. Mérida. López Rodríguez, N. (1982) Cómo evaluar textos escolares. Madrid: Cincel/Kapelusz. Martínez Henarejos, A., Ruíz Martínez, V., Luengo Jusdado, S. Y Otros (1994). Accesibilidad en el medio físico para personas con ceguera o deficiencia visual. Madrid:: ONCE.

Richadeau, F. (1976) La lisibilité. París: CEPL. 
Salvador Esteban, L. (1993) Modificaciones ambientales para deficientes visuales. En: 6 a Conferencia Internacional de Movilidad: Madrid, 9 al 12 de septiembre de 1991. Vol. I, 156-160. Madrid: ONCE.

Vázquez Menéndez, E. (1996). Políticas de integración social para personas con minusvalías. En: Actas del Congreso Estatal sobre Prestación de Servicios para Personas Ciegas y Deficientes Visuales: Madrid, septiembre de 1994, I, 301-305. Madrid: ONCE. 